\title{
ANATOMÍA DE UN SISTEMA DE INFORMACIÓN GEOGRÁFICA (SIG) PARA EL PATRIMONIO ARQUEOLÓGICO DEL CENTRO DE LA PROVINCIA DE BUENOS AIRES
}

\author{
ANATOMY OF A GEOGRAPHIC INFORMATION SYSTEM (GIS) FOR THE \\ ARCHAEOLOGICAL HERITAGE OF THE CENTER OF BUENOS AIRES \\ PROVINCE
}

\section{Carolina I. Mariano ${ }^{1}$, María Luz Endere ${ }^{2}$, Victoria Pedrotta ${ }^{3}$ y Mercedes Mariano".}

\author{
1 PATRIMONIA, INCUAPA (UE CONICET-UNICEN). Facultad de Ciencias Sociales, Universidad Nacional del \\ Centro de la Provincia de Buenos Aires. Email: caro.mariano@hotmail.com \\ 2 PATRIMONIA, INCUAPA (UE CONICET-UNICEN). Facultad de Ciencias Sociales, Universidad Nacional del \\ Centro de la Provincia de Buenos Aires. E mail: mendere@soc.unicen.edu.ar \\ 3 CONICET, Departamento de Ciencias Naturales y Antropología y Fundación Félix de Azara - Universidad \\ Maimónides. PATRIMONIA. Facultad de Ciencias Naturales y Museo, Universidad Nacional de La Plata. \\ E mail: vpedrotta@conicet.gov.ar \\ 4 PATRIMONIA, INCUAPA (UE CONICET-UNICEN). Facultad de Ciencias Sociales, Universidad Nacional del \\ Centro de la Provincia de Buenos Aires. E mail: mercedes.mariano@gmail.com
}

Presentado el: 30/01/2014 - Aceptado 12/11/2014

\begin{abstract}
Resumen
La gestión del patrimonio arqueológico en la provincia de Buenos Aires, Argentina, ha sido poco abordada en trabajos científicos pese a las numerosas investigaciones realizadas en la región durante las últimas décadas. Una de las posibles causas de la falta de políticas efectivas de protección puede deberse, entre otros factores, a la carencia de las herramientas metodológicas adecuadas. El objetivo de este trabajo es presentar un conjunto de estrategias que apuntan a resolver este problema. Las mismas se basan en el desarrollo de una metodología para la gestión de la información arqueológica de tres partidos del centro de la provincia de Buenos Aires: Tandil, Olavarría y Azul, instrumentada por medio de un Sistema de Información Geográfica que, a su vez, es susceptible de ser adaptado a cualquier otra área de interés. Se presenta la estructura interna de dicho sistema, se describe cada una de las variables de la herramienta generada y se plantea, además, la necesidad de un trabajo interdisciplinario para la obtención y sistematización de los datos. Finalmente, se brindan los argumentos por los cuales esta herramienta constituye hoy un insumo clave e innovador para la planificación de políticas de gestión patrimonial a nivel provincial.
\end{abstract}


Palabras claves: Sistema de información geográfica, Gestión del patrimonio, Provincia de Buenos Aires.

\begin{abstract}
The archaeological heritage management in Buenos Aires province, Argentina, has been scarcely analyzed in scientific works despite the great number of archaeological researches carried out in the region in recent decades. In this sense, it is proposed that one of the possible causes of the lack of effective protection policies may be due to lack of appropriate methodological tools. The purpose of this paper is to present a set of strategies that aim to solve this problem. They are based on the development of a methodology for the management of archaeological information of the municipalities of Tandil, Azul and Olavarria, located at the centre of Buenos Aires Province. It is implemented by means of a geographic information system which can be adapted to any another area of interest. The internal structure of this tool is presented, and their variables are carefully described. The need for interdisciplinary work to collect and systematize data is also stressed. Finally, the raisons why this tool constitutes an innovative and key input for planning management policies are presented.
\end{abstract}

Key words: Geographical Information System, Heritage management, Buenos Aires province.

\title{
Introducción
}

La gestión del patrimonio arqueológico es un campo dedicado a identificar, proteger y presentar al público restos materiales del pasado (González Méndez 2000; Cleere 1993; Ballart y Petit 1991, entre otros). Su desarrollo en la provincia de Buenos Aires (Argentina) es un tema que ha sido escasamente abordado en trabajos científicos hasta el momento. No obstante, se trata de una región donde se han llevado a cabo numerosas investigaciones arqueológicas a lo largo del tiempo y existe por ello un corpus de información muy amplio, generado por diversos equipos de especialistas desde hace más de dos décadas (Berón y Politis 1997; Politis y Madrid 2001; Mazzanti et al. 2002; Gradín y Oliva 2004; Martínez et al. 2004; Bayón et al. 2007; Endere y Prado 2009; Berón et al. 2010, para dar sólo algunos ejemplos de trabajos de síntesis).

Una de las posibles causas de este contraste entre el crecimiento de las investigaciones y la falta de políticas de gestión del patrimonio arqueológico en dicha provincia podría ser la carencia de metodologías de manejo del patrimonio desarrolladas por equipos interdisciplinarios, así como la falta de herramientas informáticas adecuadas para la administración de los datos disponibles. En este sentido, se considera que uno de los principales problemas a resolver es la falta de sistematización de la vasta información que se ha generado a lo largo de todos estos años desde el ámbito de la investigación arqueológica.

En este artículo se presenta una herramienta informática útil para identificar, caracterizar e inventariar el patrimonio arqueológico, que constituye una versión revisada y ampliada de un sistema inicialmente diseñado para los partidos de Tandil, Olavarría y Azul (en adelante TOA; Figura 1), de la provincia de Buenos Aires (ver Mariano 2012). Este instrumento se ha denominado SIPAB (Sistema de Información del Patrimonio Arqueológico Bonaerense), e incluye no sólo los datos cuantitativos de interés, sino también aquellas variables relacionadas con la valoración social del patrimonio regional, lo que constituye una dimensión insoslayable para su gestión (Endere y Prado 2009; Convenio Europeo del Paisaje 2000), como se verá más adelante. 


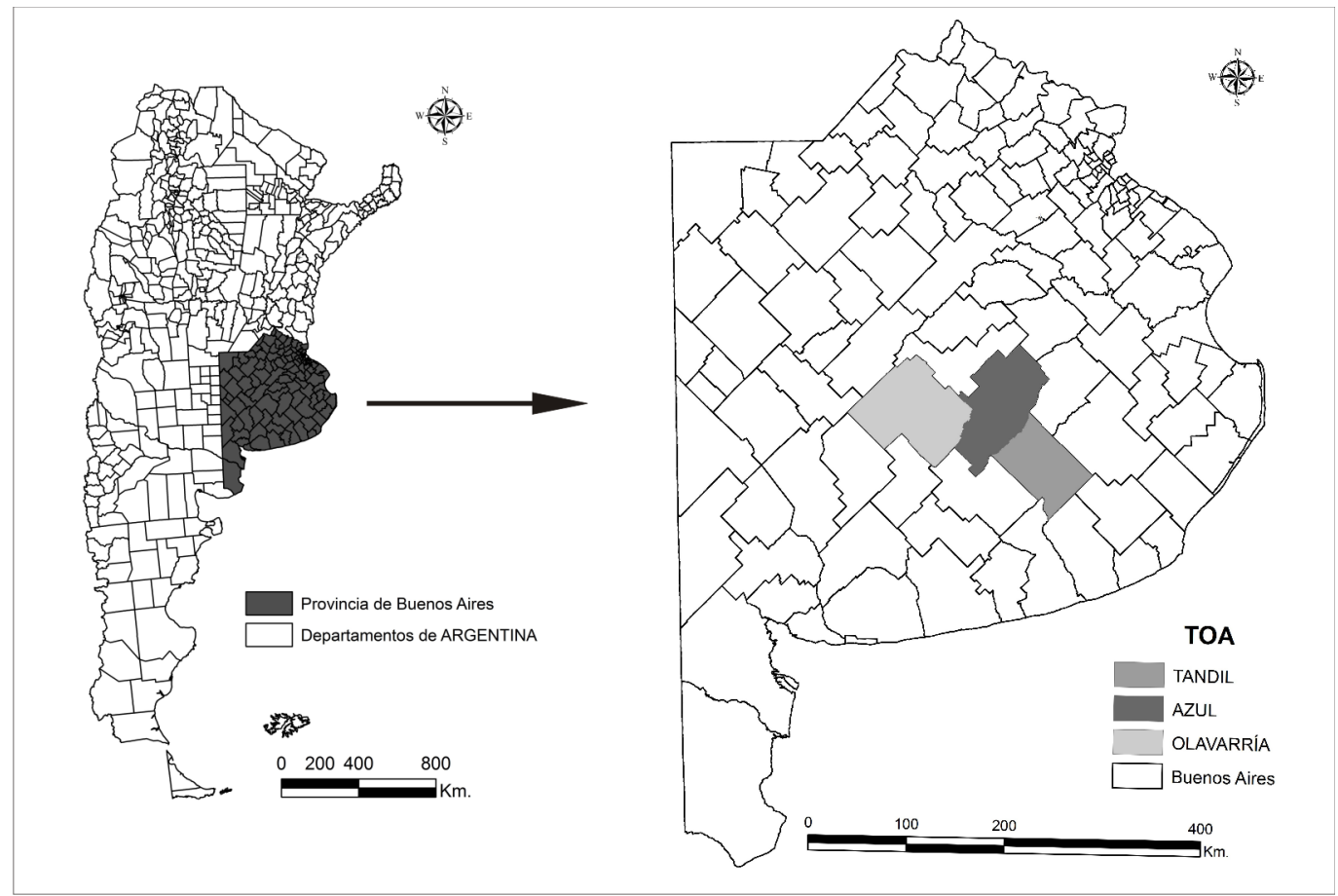

Figura 1. Ubicación de los tres partidos del centro de la provincia de Buenos Aires.

Este artículo tiene la finalidad de presentar cada uno de los campos que componen el $\mathrm{SIG}^{1}$, comenzando por la descripción minuciosa de las variables incluidas en la llamada "Planilla de Datos Madre" (en adelante PDM). Esta planilla base permite sistematizar u ordenar estructuradamente una gran cantidad de información que es a priori difícil de conciliar, ya sea porque ha sido producida por múltiples investigadores a lo largo del tiempo, todos ellos guiados por paradigmas diferentes y/o por las disímiles metodologías de investigación empleadas.

La descripción de los atributos, así como el valor científico de cada sitio arqueológico, se relevaron a partir de la información que figura en los trabajos publicados sobre ellos, tanto nacionales como internacionales. También se consideró necesario incorporar la información producida en el marco de estudios de impacto arqueológico (la llamada "literatura gris"), aún cuando se trate de informes no publicados, para lo cual se contó con la autorización de sus autores.

Para el estudio de la valoración social de los sitios fue necesario llevar adelante trabajos de campo empleando metodología etnográfica (Guber 2004) con diferentes representantes de las comunidades locales, con el objeto de indagar acerca de su significación cultural. Posteriormente se establecieron criterios a fin de poder ingresar esa información de tipo cualitativa a la PDM (Mariano 2012, ver también Mariano et al. 2013).

Para armar el diseño definitivo de la planilla de datos madre fueron necesarias numerosas pruebas y rediseños, lo que permitió que el ingreso de los datos fuera, paulatinamente, cada vez más ajustado. De esta manera, se pretendió desarrollar una 
metodología para sistematizar la información referente al registro arqueológico del área que incluyera, además, todos aquellos datos de carácter administrativo y técnico-científico que pudieran resultar relevantes.

\section{Descripción de los campos de la Planilla de Datos Madre}

El conjunto de variables que se detallan a continuación constituye la "anatomía" del SI$\mathrm{PAB}$. Se parte de asumir que toda la información arqueológica puede delimitarse en entidades discretas (campos o columnas) y que, así como el proceso de producción de información es sumamente crítico, también lo es el modo en que ésta se estructura dentro de un sistema de información. A su vez, se generaron campos para el ingreso de datos cualitativos, de modo que fuera posible interactuar con ambos tipos de información simultáneamente en función de los problemas que pudieran llegar a presentarse para los operadores.

Una vez que el contenido total de la PDM se vuelca al software elegido, puede hacerse uso de las herramientas disponibles para el procesamiento de la información. Es importante aclarar aquí que la eficacia en el uso de instrumentos informáticos no depende exclusivamente del programa que se elige sino, fundamentalmente, de toda la planificación, estructuración y relevamiento de los datos de los que se compone una base. Esto es lo que realmente garantiza la calidad de la herramienta de gestión.

Las variables y el modo en que los campos codifican en el SIPAB

\section{Nominación e inventariado}

- Número de inventario del sitio

Este es un número entero que va a identificar a cada sitio/entidad patrimonial. La base de datos se elabora en planillas de cálculo del programa Excel (Microsoft Office), ya que constituye un formato capaz de almacenar gran cantidad de información y es fácilmente transferible a cualquier Sistema de Información Geográfica.

\section{- Nominación del sitio arqueológico/entidad patrimonial}

Es el nombre que le han dado los investigadores a cada sitio/ entidad patrimonial. Se codifican con el mismo número de inventario de la PDM.

\section{- $\underline{\text { Sigla }}$}

Se utiliza para abreviar el nombre del sitio/entidad patrimonial. No deberá ser igual para dos sitios diferentes.

\section{- Localidad arqueológica}

Las localidades arqueológicas están constituidas por áreas que contienen varios sitios arqueológicos o entidades patrimoniales relacionadas. Representan, por ello, puntos del espacio con concentraciones diferenciales de restos o materiales culturales, lo que estaría indicando que su utilización humana fue relativamente intensiva. Para su codificación se coloca el nombre (como texto), y se deja el campo sin información cuando la entidad no forma parte de una localidad arqueológica. 


\section{Datos del investigador}

- Investigador a cargo del sitio

En este campo se indica el nombre del investigador a cargo de ese sitio en la actualidad, ya que puede haber habido varias etapas de investigación con diferentes investigadores a cargo a lo largo del tiempo.

- Filiación institucional del investigador a cargo en la actualidad

Se refiere a la institución en la que trabaja el investigador a cargo de cada sitio/entidad patrimonial. Es conveniente incorporar una lista control de abreviaturas.

- Dirección de correo electrónico del investigador a cargo

Esta información posibilita la comunicación entre el investigador responsable y cualquier interesado en la entidad patrimonial.

\section{Funcionalidad y adscripción cultural de los sitios}

\section{- $\underline{\text { Tipo de sitio }}$}

Este campo asigna las funciones que los investigadores han establecido para cada entidad patrimonial, evitando preferentemente la consideración de funcionalidades secundarias (Ladrón de Guevara 2011). Se codifican con el número 1 (uno) los casos en que la funcionalidad fue asignada al sitio en cuestión, y con 0 (cero), los casos en que el sitio/ entidad no presenta dicha funcionalidad. Si no cabe duda de que un sitio haya tenido más de una función, se coloca el número 1 en más de un campo.

* Asentamiento residencial

Sitios arqueológicos con evidencia de actividades múltiples producidas por ocupaciones humanas por períodos relativamente prolongados o permanentes. Los materiales hallados pueden indicar habitación humana redundante, prácticas relacionadas a la preparación y consumo de alimentos, la confección de instrumentos líticos, la elaboración de cerámica, etcétera.

* Asentamiento temporario

Se trata de sitios arqueológicos con evidencia de ocupaciones breves. Sus restos materiales pueden indicar que se usaron como lugares de habitación, prácticas relacionadas a la preparación y consumo de alimentos, entre otras actividades.

* Cantera

Se trata de sitios arqueológicos que evidencian haber sido escenario de actividades de extracción y/o de las primeras etapas de reducción de materias primas líticas. Estas se encuentran naturalmente disponibles en el lugar de hallazgo.

* Cacería

Se trata de sitios arqueológicos con evidencia material de actividades relacionadas a la caza de animales y/o a las primeras etapas de su procesamiento.

\section{* Con Carácter Ritual}

Son sitios con evidencia material que permite inferir que las actividades llevadas a cabo en ese lugar poseyeron un componente simbólico preponderante. 
* Funerario

Son aquellos sitios que presentan restos óseos humanos intencionalmente enterrados.

* Almacenaje

Se trata de sitios acondicionados para almacenar diversos productos, tanto elaborados como materias primas.

* Comercio/ Intercambio

Se trata de lugares en los cuales se llevaron a cabo actividades comerciales, tales como el trueque e intercambio de diversos productos, transacciones monetarias, etc. En el área de estudio pueden ser tanto ferias -espacios que congregaban periódicamente a diversos grupos sociales y étnicos para intercambiar y/o comerciar productos- como lugares más discretos, usualmente con instalaciones edilicias, que funcionaban de modo permanente - típicamente, las "pulperías".

\section{* Punto de Avistaje}

Se trata de sitios que, sea por su ubicación topográfica y/o por el acondicionamiento humano que presentan, denotan haber sido usados para vigilar los espacios circundantes.

* Construcción defensiva

Usualmente son fortificaciones situadas en lugares de frontera, que cumplían una función defensiva y de avance sobre las tierras ocupadas por los grupos indígenas. Generalmente presentan restos de algún tipo de edificación militar y materiales que corresponden a diferentes momentos del siglo XIX, evidenciando un proceso de contacto entre los pueblos originarios y la sociedad hispano-criolla, caracterizado tanto por procesos de tensión y conflicto por la posesión y el control de la tierra, como por el desarrollo de mecanismos de intercambio. Pueden clasificarse, según su tamaño, en fuertes y fortines, entre otros.

* Muros de piedra aislados

También se trata de edificaciones construidas mediante el encastre de bloques de piedra, sin utilizar argamasa o mortero. A diferencia de los recintos, estos no circunscriben un área sino que se extienden en forma longitudinal "desde decenas hasta varios cientos de metros" (Pedrotta 2005: 389).

* Camino/rastrillada

Las rastrilladas eran antiguos caminos o rutas utilizados habitualmente por los grupos indígenas y luego por la población hispano-criolla. Estos caminos unían puntos estratégicos dentro del paisaje. Desde el punto de vista económico constituían rutas óptimas para sortear los obstáculos geomorfológicos del territorio pero, además, estos caminos "pueden considerarse como un producto cultural y, en consecuencia, visualizarse como una expresión material de la construcción social del paisaje" (Curtoni 2007: 268). Pueden clasificarse en "principales" y "secundarios" (Trombold 1991) en función de su ancho y longitud de la conexión ${ }^{3}$. Debido al gran impacto de la agricultura, no siempre pueden distinguirse a simple vista. En la base de datos van a registrarse con tres puntos (coordenadas geográficas) diferentes, de modo que la unión de los tres, por medio de una línea, señalice la rastrillada. En la PDM, los tres puntos se ingresan con el mismo número de inventario porque se trata de una entidad arqueológica única.

* Ganadería y pastoreo

Refiere a sitios vinculados a la producción ganadera y/o pastoril. En la zona de 
estudio, generalmente se trata de edificaciones construidas con la técnica de pirca seca (paredes hechas mediante el encastre de bloques de piedra, sin utilizar argamasa o mortero), que delimitan espacios relativamente cerrados de formas y dimensiones muy variadas (Pedrotta 2009).

* Producción agrícola

Refiere a sitios vinculados a la producción agrícola. Puede tratarse de campos de cultivo, obras hidráulicas, entre otros.

* Molino harinero

Se trata de construcciones edilicias (o los restos de ellas) que constituyen sitios patrimoniales por cuanto la evidencia material (cronológicamente superior a los 100 años de antigüedad) permite analizar la funcionalidad que tenían en el pasado. Una particularidad de estos sitios es su relativa cercanía temporal con la actualidad, lo cual genera vínculos identitarios con ciertos sectores de la sociedad que favorecen su salvaguarda (i.e. el sitio El Molino Viejo, construido en el año 1881 en el partido de Olavarría).

* Horno calero (Arqueología Industrial)

Son construcciones (o los restos de ellas) de carácter industrial utilizadas para la producción de cal, empleando tecnologías de calcinación en las que se cocinan materias primas minerales como la piedra caliza o la dolomita (Paz 2009, 2012).

* Indeterminado

Aún no se ha podido identificar la funcionalidad del sitio.

\section{- Adscripción cultural del sitio}

Este campo asigna la adscripción cultural que los investigadores han establecido para cada entidad patrimonial. Se codifican con el número 1 (uno) los casos en que la atribución cultural fue asignada al sitio en cuestión, y con 0 (cero) los casos en que el sitio/entidad no presenta dicha adscripción. Si no cabe duda de que el sitio corresponde a más de una adscripción cultural, se coloca el número 1 en más de un campo.

* Indígena

Refiere a los pueblos originarios del territorio americano.

* Hispano-criolla

Refiere tanto a la población originaria de la península ibérica que se instaló desde el siglo XVI como consecuencia de la conquista española como a la población criolla nacida posteriormente en el actual territorio de Argentina.

* Inmigración europea

Refiere a la población procedente de diversos países europeos que migró masivamente a Argentina a partir del último cuarto del siglo XIX y las primeras décadas del XX.

* Mixta/Mestiza

Corresponde a una combinación o mezcla de las adscripciones culturales anteriores, no diferenciables por medio del estudio arqueológico. 
* Indeterminada

Aún no se ha podido identificar

4. Fecha de descubrimiento e investigación

- Fecha de descubrimiento del sitio

Refiere al año de descubrimiento del sitio o la localidad arqueológica. Se codifica con un número entero de cuatro cifras (e.g. 1998).

\section{- Fecha probable de emplazamiento del sitio}

En aquellos casos en los que se haya documentado la fecha exacta de origen/construcción/levantamiento, esa será la información que se vuelque en relación a esta variable (i.e., fuertes, fortines, hornos caleros, edificios, etc.). Se codifica con el número entero de cuatro cifras. Puede ocurrir que no se conozca certeramente la fecha exacta de emplazamiento de la entidad arqueológica, o bien, que diferentes investigadores no acuerden en este aspecto en particular. Por tal razón, se abrirá un campo paralelo para colocar la fecha posible de emplazamiento del sitio.

\section{- Etapas de investigación del sitio}

Los sitios arqueológicos pueden haber pasado por diferentes etapas de investigación a lo largo del tiempo y todas ellas deben quedar registradas en la PDM. Por ejemplo:

* Etapa de investigación 1: Se coloca el año de inicio y el nombre del investigador a cargo en ese momento.

- Publicaciones relacionadas

Esta información se ingresa en un apéndice referenciado (por medio de un link) al $\mathrm{N}^{\circ}$ de inventario de cada sitio. Consiste en una serie de planillas en las cuales figuran todas las referencias bibliográficas que refieren a cada sitio/entidad patrimonial específicamente, como así también citas relacionadas al área en el que éste se emplaza y su temática particular. Este apéndice digital forma parte de la PDM.

\section{Localización}

\section{- Coordenadas geográficas}

Los sistemas de coordenadas permiten establecer una relación entre los datos de un software SIG y su posición en el mundo real, proceso que se conoce como georreferenciación. En este sentido, se distinguen dos tipos diferentes:

- Sistemas de Coordenadas Geográficas: que son útiles para identificar las posiciones de los puntos en cualquier lugar de la superficie de la Tierra. El más común es el sistema de coordenadas esféricas (Latitud/Longitud), que fue el utilizado a la hora de generar el SIPAB. La latitud y longitud son ángulos que se miden desde el centro de la tierra en relación al plano del Ecuador y al plano del Meridiano de Greenwich, respectivamente. 
- Sistemas de Coordenadas Planares o Cartesianas: Se basan en la proyección de los puntos sobre una superficie plana, donde las posiciones son identificadas por coordenadas $\mathrm{X}, \mathrm{Y}$ respecto del punto marcado como origen. A estas dos coordenadas que marcan la posición horizontal, se añade una tercera para la posición vertical.

A su vez, el datum horizontal es un marco de referencia utilizado para ubicar elementos en la superficie de la Tierra. Está definido por un esferoide y la posición del esferoide con relación a la superficie. El datum que se utilizó en el SIPAB es el WGS84 (del inglés World Geodetic System 1984, que significa Sistema Geodésico Mundial).

Se identifican el par o conjunto de pares de coordenadas $(X, Y)$ que definen espacialmente una entidad geométrica -punto, línea o polígono- (Ladrón de Guevara 2011: 66). A tal efecto se indican Latitud Sur, Longitud Oeste y, para definir un área, se señala el Polígono.

\section{- Partido}

En Argentina, se denomina partido al territorio de una jurisdicción o administración que tiene por cabeza un pueblo principal. Este campo se completa con números enteros, cada uno asignado a un partido del área de interés.

\section{- $\underline{\text { Localidad }}$}

Se trata de una división territorial o administrativa con identidad propia. En general, constituyen núcleos de pequeño tamaño y escasa cantidad de habitantes.

\section{- Altura relativa respecto del nivel del mar}

Es la distancia vertical de un punto de la Tierra respecto al nivel del mar (cero). Se mide en metros. Este campo se llena con un número entero.

\section{- Superficie aproximada del sitio}

Permite señalar el área estimada que ocupa el sitio/entidad patrimonial. Se mide en metros cuadrados. Este campo se llena con un número entero.

\section{Datación}

\section{- Cronología}

Para ingresar los datos referentes a la cronología de las entidades arqueológicas se generan todos los campos detallados abajo. Codifican con el número 1 (uno) los casos positivos y con el 0 (cero) los casos negativos.

* Sin datación disponible

Se refiere a los casos en que ningún hallazgo ha podido ser ubicado cronológicamente todavía.

* Holoceno Temprano

Corresponde a aquellos sitios que han podido ser ubicados cronológicamente entre los 12.000 y 7.000 AP. 
* Holoceno Medio

Corresponde a aquellos sitios que han podido ser ubicados cronológicamente entre los entre 7.000 y $3.000 \mathrm{AP}$.

* Holoceno Tardío

Corresponde a aquellos sitios que han podido ser ubicados cronológicamente entre los entre 3.000 años antes del presente y el inicio del período Post-hispánico en el área de estudio. Convencionalmente, se toma al siglo XVI como criterio, aunque las evidencias fehacientes de contacto en la región sean algo posteriores.

* Período Colonial

Corresponde a aquellos sitios que han podido ser ubicados cronológicamente en el período comprendido entre los años posteriores a la conquista española del territorio y el final del dominio colonial, que convencionalmente se sitúa en 1810 . Se ingresan como años después de Cristo (en adelante DC).

* Período Republicano

Corresponde a aquellos sitios que datan del período comprendido entre la independencia de España -tomando convencionalmente la fecha de 1810- y los 100 años antes del presente que marca la legislación como límite para que un sitio o entidad sean considerados patrimonio arqueológico (ver Ley 25.743 y su Decreto Reglamentario; Endere y Prado 2009). Se ingresan como años después de Cristo (en adelante DC).

\section{- Material a partir del cual se obtuvo el fechado}

Este campo especifica cuál fue la fuente material que posibilitó ubicar cronológicamente el sitio/entidad patrimonial. Por no tratarse de variables excluyentes entre sí, cada campo codifica con un número diferente. Debido a que la cronología del sitio podría haberse obtenido por medio del análisis de más de una variable de las que se proponen en la lista control, en la PDM se abren dos campos idénticos (o más, si fuera necesario) para su llenado.

* Muestra de material óseo o dentario

* Muestra de carbón

En el caso de los fechados radiocarbónicos, hay que tener en cuenta la necesidad de un ajuste o calibración radiocarbónica, ya que existe un desequilibrio cronológico en los niveles de 14C atmosféricos, que no fueron constantes a lo largo del tiempo, por lo que, para su mayor exactitud necesitan de una calibración que pueda subsanar las alteraciones atmosféricas de su tasa histórica. Para esto, el método usado consiste en establecer una comparación entre dos edades cronológicas obtenidas sobre el mismo objeto, una corresponde al método del 14C y otra determinada por medio de una técnica más precisa.

\section{* Vidrio}

Generalmente es posible ubicar temporalmente materiales vítreos gracias a su proceso de manufactura. Además, algunos de ellos contienen sellos o inscripciones que pueden indicar tanto la procedencia como el año de elaboración.

* Fuente documental escrita

Son fuentes de información cuyo mensaje queda almacenado en un documento o soporte físico (generalmente papel). 
* Cerámica

Este grupo incluye objetos de porcelana, loza, gres y barro cocido. Su datación es similar a la del vidrio, por medio de evidencias asociadas al proceso de manufactura y/o la presencia de sellos, grabados, inscripciones.

* Instrumentos de metal

La composición y características del proceso de elaboración de los objetos de metal permiten, en algunos casos, estimar las técnicas y la fecha de su confección.

* Materia orgánica del suelo

El fechado se ha realizado mediante el análisis de una muestra de suelo

* Asociación

En este caso, el fechado relativo no se obtuvo por medio del análisis de un material concreto, sino por la asociación que puede establecerse en función de otros sitios de características muy similares. Por ejemplo, algunos corrales de piedra han sido datados y otros no, sin embargo, podría inferirse que esas estructuras corresponden a un período temporal cercano al de aquellos sitios para los cuales se cuenta con información cronológica.

* Otros

Cualquier otro tipo de material no incluido en esta lista control, a partir del cual se haya podido datar un sitio o entidad patrimonial, deberá ser ingresado en este campo sin codificación, para poder ser incluido al sistema posteriormente.

\section{Condiciones del emplazamiento}

\section{- Geoforma del emplazamiento}

Las geoformas son cuerpos tridimensionales que tienen forma, tamaño, volumen y topografía, todos ellos elementos que generan un relieve determinado.

* Cerro

Se trata de geoformas de relieve positivo, de escasa altura relativa respecto del nivel del mar (entre 100 y 500 metros aproximadamente). En este campo codifican con el número 1 (uno).

* Llanura

Se refiere a geoformas con poca o ninguna variación de altura en su superficie respecto al nivel del mar. En este campo codifican con el número 2 (dos).

* Planicie de inundación

Son las áreas adyacentes a ríos, arroyos o lagunas, que se encuentran sometidas a recurrentes inundaciones debido a la crecida de dichos cuerpos hídricos. En este campo codifican con el número 3 (tres).

* Otros

Se ingresan sin código, colocando el nombre de la geoforma correspondiente para luego incluir la nueva variable en el sistema. 
- Nominación de la fuente de agua dulce más cercana

En el área del TOA se trata de arroyos, lagunas y manantiales. En otras áreas deberán incluirse ríos, glaciares, etcétera. Este campo se completa alfabéticamente, escribiendo el nombre de la fuente de agua más cercana al sitio arqueológico.

- Distancia aproximada del sitio a la fuente de agua dulce más cercana

Es la distancia que existe entre el sitio/entidad arqueológica y la fuente de agua dulce más cercana, medida en metros lineales entre una y otra. La cercanía de las fuentes de agua es un tema importante para la gestión del patrimonio cultural en el centro bonaerense, debido a las frecuentes inundaciones que sufre la región. Por ello constituye un factor de riesgo significativo a la hora de juzgar la vulnerabilidad de los sitios. arqueológicos. Este campo se llena con un número entero aproximado.

\section{- $\underline{\text { Posición de los materiales hallados }}$}

En este campo se codifican con número 1 (uno) los casos positivos y con número 0 (cero) los casos negativos. Las variables se despliegan en campos diferentes debido a la posibilidad de que un mismo sitio/entidad haya presentado materiales en diferentes posiciones de hallazgo. El campo de información se organiza de este modo porque se trata de variables no excluyentes entre sí.

* A cielo abierto en superficie

Los materiales se encuentran en exposición subaérea.

* A cielo abierto en estratigrafía

Los materiales se hallaron enterrados o semienterrados y su recuperación es producto de excavaciones arqueológicas.

* Abrigo rocoso en superficie

Los materiales arqueológicos son hallados en superficie, ya sea en la boca o en el interior de un alero de piedra.

* Abrigo rocoso en estratigrafía

Los materiales arqueológicos son hallados en estratigrafía, ya sea en la boca o en el interior de un alero de piedra.

* Sumergido

El sitio y los materiales arqueológicos se encuentran bajo las aguas.

\section{- Cartografía}

En este apartado se consigna el material cartográfico disponible con respecto al sitio/entidad patrimonial. Puede tratarse de hojas topográficas del Instituto Geográfico Nacional (IGN), cartas cuya base cartográfica representa sobre un plano todos los accidentes naturales y artificiales de un área determinada. Las más comunes están elaboradas en las escalas 1:50.000 ó 1:100.000.

Asimismo pueden incluirse fotogramas aéreos del Departamento de Aerofotogrametría del Ministerio de Infraestructura, Obras y Servicios Públicos de la Provincia de Buenos Aires. También imágenes satelitales o en cualquier otro tipo de cartografía de los múltiples sitios de internet que permiten el acceso a bases permanentes GPS. 


\section{Condiciones actuales del sitio}

\section{- Estado de conservación}

El estado de conservación de un sitio arqueológico tiene que ver directamente con la probabilidad que éste tenga o no de seguir existiendo. En términos de la gestión patrimonial, la información que se pueda recabar en relación a esta variable es de vital importancia para la aplicación de criterios de selección y de medidas de protección.

* Muy bueno

Su estado de conservación es estable, sin señales de deterioro. La entidad patrimonial se encuentra bajo la protección/cuidado informal de algún ente privado o público. Codifican con el número 4 (cuatro).

* Bueno

Con signos de deterioro leve por causas naturales y/o antrópicas pero con protección/ cuidado informal de algún ente privado o público. Codifican con el número 3 (tres).

* Regular

Con signos de deterioro por causas naturales y/o antrópicas y sin protección/cuidado de ningún ente privado o público. Codifican con el número 2 (dos).

* Muy deteriorado

Presenta alto grado de vulnerabilidad y su pérdida total es inminente si no se toman medidas urgentes de protección. En estos casos, es conveniente realizar un registro completo (o conservación mediante registro) para evitar la pérdida de información. Codifican con el número 1 (uno).

* Destruido

Destruido por causas naturales y/o antrópicas (vandalismo, excavación arqueológica total, obras de infraestructura y/o remoción de suelos, etcétera). En los casos en que las variables de destrucción puedan detectarse o controlarse, será conveniente realizar un rescate arqueológico que minimice la pérdida total de información.

Puede suceder que la entidad arqueológica se haya "preservado mediante registro", es decir, el sitio ya no tiene existencia material pero se cuenta con la información producida mediante una investigación arqueológica y/o existen los materiales procedentes de dicha excavación. En este caso, la base de datos estaría funcionando como depósito virtual de ese patrimonio. Codifican con el número 0 (cero).

\section{- Depósito de las colecciones}

Informa sobre el nombre de la Institución y el espacio físico en el que se encuentran depositadas las colecciones arqueológicas. Cuando se trate de evidencia material inmueble (i.e. arte rupestre, estructuras construidas, concheros, etc.), se colocará "in situ" en el campo correspondiente. Cuando se trate de evidencia material mueble, se escribirá el nombre de la entidad depositaria (i.e. Museo Dámaso Arce, Depósito INCUAPA, etcétera) y los datos de la institución.

* Institución 
* Provincia

* Ciudad

* Calle y número

* Teléfono

* Página web o correo electrónico de la entidad

* In Situ

\section{- Protección legal específica}

* Protección legal específica Nula

No existe una norma que proteja específicamente al sitio/entidad patrimonial además de la normativa general de patrimonio a nivel nacional: ley 25.743. Codifican con el número 0 (cero).

* Protección específica por norma municipal (ordenanza o decreto)

Además de la normativa general de patrimonio, existe una norma del municipio que protege la entidad arqueológica. Codifican con el número 1 (uno).

* Protección específica por ley provincial

Existe una declaratoria especial por ley provincial (i.e. Bien Cultural de Interés Provincial; Áreas Naturales Protegidas cuya normativa involucra a los Sitios Arqueológicos) (Ver Endere 2009: 38 y 39). Codifican con el número 2 (dos).

* Protección específica por ley nacional

Además de la normativa general de patrimonio, existe una declaratoria especial por ley nacional (i.e. Monumentos o Lugares Históricos Nacionales). Codifican con el número 3 (tres).

* Declaratoria UNESCO

La entidad arqueológica recibe el reconocimiento y protección de la Organización de las Naciones Unidas. Codifican con el número 4 (cuatro).

\section{- $\underline{\text { Potencial turístico }}$}

Se tiene en cuenta la combinación de una serie de condiciones que se detallan a continuación. Para posicionar a una entidad patrimonial en una categoría dada, será necesario que esta reúna, al menos, seis de las condiciones detalladas. Muchas de estas variables son susceptibles de modificarse a lo largo del tiempo, por lo que una entidad podría cambiar de categoría en el futuro.

* Alto potencial turístico

- El sitio/entidad patrimonial resulta atractivo para el público general, ya que representa un testimonio material de momentos significativos de la historia regional.

- Su estado de conservación fue determinado como "bueno" o "muy bueno".

- Se encuentra dentro de un radio máximo de 10 kilómetros con respecto a un centro poblado.

- Existen rutas de acceso al sitio para cualquier tipo de vehículo.

- Se cuenta con los permisos correspondientes para su visita (i.e., de los propietarios legales del terreno e investigadores a cargo del estudio del sitio/entidad).

- Se proyectan planes de manejo adecuados, realizados bajo la dirección de arqueólogos especializados para el aprovechamiento de este tipo de recursos culturales, que incorporan la opinión de todos los grupos de interés involucrados.

- Se cuenta con un espacio físico para la construcción de instalaciones sanitarias, para descanso y servicio de comida (González Méndez 2000). Codifican con el número 3 (tres). 
* Potencial turístico medio

- El sitio/entidad patrimonial resulta atractivo para el público general, en tanto testimonio material de acontecimientos transcurridos durante la historia del área.

- Su estado de conservación fue determinado como "bueno" o "muy bueno".

- Se encuentra dentro de un radio máximo de 25 kilómetros con respecto a un centro poblado.

- El acceso al sitio sólo es posible mediante el uso de vehículos especiales o a pie.

- Se cuenta con los permisos correspondientes para su visita (léase: de los propietarios legales del terreno e investigadores a cargo del estudio del sitio/entidad).

- Se proyectan planes de manejo adecuados, realizados bajo la dirección de arqueólogos y/o personas capacitadas, para el aprovechamiento de este tipo de recurso cultural, incorporando la opinión de todos los grupos de interés involucrados.

- Debería haber un espacio físico suficiente para ser destinado, al menos, para la construcción de instalaciones sanitarias adecuadas. Codifican con el número 2 (dos).

* Potencial turístico bajo

- El sitio/entidad patrimonial resulta escasamente atractivo para el público general, ya que los materiales arqueológicos han sido extraídos y trasladados a otro lugar.

- Su estado de conservación fue determinado como "bueno" o "regular".

- Se encuentra dentro de un radio mayor a los 35 kilómetros con respecto a un centro poblado.

- El acceso no es difícil en vehículos especiales, pero sólo es posible llegar a pie hasta el sitio propiamente dicho.

- Se cuenta con los permisos correspondientes para su visita (i.e. de los propietarios legales del terreno e investigadores a cargo del estudio del sitio/entidad).

- Se proyectan planes de manejo adecuados, realizados bajo la dirección de arqueólogos y/o personas capacitadas, para el aprovechamiento de este tipo de recurso cultural. - Los planes de manejo incorporarán la opinión de todos los grupos de interés involucrados.

- No existe la posibilidad o el interés de construir un refugio con instalaciones sanitarias. Codifican con el número 1 (uno).

* Nulo o muy bajo

- El sitio carece de visibilidad.

- Su estado de conservación fue determinado como "malo" o "destruido".

- Ha desaparecido (por causas diversas).

- El lugar de emplazamiento es inaccesible.

- El sitio no resulta atractivo para el público general.

- Codifican con el número 0 (cero).

Como ya se señaló, en algunos casos, estas características podrían cambiar con el tiempo $\mathrm{y}$, por lo tanto, se podría reclasificar el sitio cuando fuera oportuno.

\section{- $\underline{\text { Potencial arqueológico }}$}

Referido a aquellos casos en que la entidad patrimonial y sus inmediaciones tienen potencialidad de contener más evidencias arqueológicas de las que ya han sido descubiertas, investigadas y publicadas. También se incluyen las áreas que, a pesar de no haber sido exploradas todavía, tienen alto potencial de contener registro arqueológico (i.e., las cercanías de cursos de agua permanentes o los lugares por donde se extendían las "rastrilladas", etcétera. 
Las entidades con potencialidad arqueológica codifican con el número 1 (uno).

Las entidades para las cuales no se estime potencialidad arqueológica codifican con el número 0 (cero).

\section{- Uso actual del suelo}

Se refiere a los diferentes tipos de actividades productivas u ocupación actual que están realizándose en las inmediaciones del sitio o la localidad arqueológica y, eventualmente, generando impacto sobre la entidad o sobre su zona de amortiguamiento o buffer (Ladrón de Guevara 2011: 53).

* Agricultura y ganadería

* Espacio urbanizado

* Minería

* Camino/ruta/vía férrea

* Turismo

* Museo de sitio

* Industria

* Otras actividades socioculturales.

9. Registro arqueológico presente en los sitios

\section{- $\underline{\text { Hallazgos }}$}

Cuando en las publicaciones disponibles se documenten y describan las características de los hallazgos de cada sitio/entidad patrimonial, se volcarán todos esos datos en términos de presencia/ausencia/falta de información. La presencia se codifica en todos los casos con el número 1 (uno), la ausencia con el número 0 (cero) y la falta de información con el número 2 (dos).

* Elementos arquitectónicos de pirca seca

*Elementos arquitectónicos de otros materiales (cimientos, paredes de ladrillo, pisos, etc.)

* Artefactos líticos

* Material óseo de aves

* Material óseo de fauna terrestre

* Material óseo de fauna acuática

* Material óseo humano

* Material óseo de fauna extinta (en caso de existir fauna extinta, en el campo siguiente se coloca la nominación científica de la especie representada (datos alfabéticos). Si hay más de una especie extinta en un mismo sitio, se abrirán campos paralelos indicándose: “Especie extinta 1", "Especie extinta 2", etcétera.

* Material malacológico acuidulce

* Material malacológico marino

* Carbón

* Pinturas rupestres

* Ocres y pigmentos

* Cerámica

* Ladrillo

* Loza

* Artefacto de hueso

* Vidrio 
* Metal

* Restos vegetales

* Textiles

* Otro

\section{$\underline{\text { 10. Identificación de los criterios de valoración }}$}

Cuando una entidad arqueológica es reconocida por un grupo u organización social, se registra en la base de datos cuál es el tipo de valoración de la que goza. Para esto, se abren los 10 últimos campos (o columnas) que conforman la PDM y se describen a continuación:

$1^{\circ}$ Desde el punto de vista científico los criterios a tener en cuenta son los siguientes:

\section{Rareza}

Este criterio refiere a aquellas entidades arqueológicas que poseen un tipo de registro que sale del patrón recurrente para el área en cuestión (i.e., los sitios de la localidad arqueológica "Sierras de Curicó" evidencian las únicas pinturas rupestres reportadas en el TOA). Sin embargo, se considera que también deben protegerse aquellos sitios que presenten todas o la mayoría de las características típicas, y no sólo los que rompen el patrón (i.e. las canteras serranas con evidencia de explotación asidua de materias primas líticas).

Vulnerabilidad

Se refiere a los sitios arqueológicos que puedan verse afectados por diferentes actividades que se desarrollen en el área de su emplazamiento. Particularmente, en el TOA se asocian a zonas en las que se practican intensivamente la agricultura, la ganadería y la minería, donde podría privilegiarse la explotación económica en detrimento del valor y la significación arqueológica del patrimonio contenido en ellas. Aquellos sitios que se consideren con alto potencial arqueológico y/o valoración social deberían ser protegidos de un modo diferencial, puesto que están expuestos a daños irreversibles y, por ende, a la pérdida tanto de ese patrimonio como de la información que podría proporcionar su estudio.

Fuentes históricas asociadas

La significación o relevancia histórica de un sitio/entidad arqueológica puede estar dada por la existencia de fuentes documentales y registros de antiguas menciones relacionadas al mismo. En el apéndice de citas bibliográficas de cada uno de los sitios ingresados a PDM, deberán encontrarse también citados estos antiguos trabajos, de modo que puedan consultarse de modo directo cuando el usuario lo requiera.

\section{Grupo de valor}

Se trata de sitios o entidades arqueológicas con diferentes grados de significación, que se encuentran asociados de algún modo (por poseer características similares, por cercanía geográfica o cronológica, en función de la similitud de sus hallazgos, etcétera). En estos casos, se propone preferentemente la protección del "grupo de sitios" (e.g. PPG16 2006) en conjunto, en vez de hacerlo aisladamente. Un ejemplo para el área central de la provincia de Buenos Aires lo constituyen la localidad arqueológica "Sierras del Curicó", en Olavarría y los "corrales de piedra" de los partidos de Azul y Tandil, que gozan ambos de alta valoración científica. También el conjunto de hornos caleros de las localidades serranas de Olavarría, que evidencian alta valoración social (ver Paz 2012).

$2^{\circ}$ Desde el punto de vista social se seleccionaron aquellas entidades arqueológicas que poseen: 
Valor identitario

Se establecerá cuando haya quedado demostrado, por medio del trabajo de campo antropológico, que ese patrimonio es valorado por los miembros de una comunidad local. El valor identitario está constituido por aquellas características que contribuyen a la definición de las identidades de poblaciones de diferente origen étnico, geográfico, religioso, etc., que habitan una región. En relación a este punto, se ha propuesto que los sitios arqueológicos son lugares que pueden ayudar tanto a comprender el pasado como a enriquecer el presente (Hall y Mc Arthur 1996).

- Los sitios que gozan de este tipo de valoración social codifican con el número 1 (uno).

- Los sitios para los cuales no se identifique este tipo de valoración codifican con el número 0 (cero).

Valor educativo

Los sitios patrimoniales proveen de experiencias "en vivo" acerca de la historia de un lugar (Hall y Mc Arthur 1996). En este sentido, Mark (1996) propone que "la historia es un relato desarticulado" que la arqueología y el patrimonio pueden ayudar a articular, para producir interpretaciones más complejas y comprenderla mejor. En general, se advierte que los espacios de educación no formal por excelencia son los museos locales, pero se considera importante relevar la información acerca de cuáles de todos los sitios arqueológicos listados en el sistema de información de un área determinada serían susceptibles de ser utilizados con fines educativos. La ciudadanía puede aprender diferentes aspectos del pasado, democratizándose y socializándose así el proceso de la investigación científica.

Para recibir este tipo de valoración deberían darse ciertas condiciones mínimas y fundamentales:

a) cercanía relativa a un lugar poblado,

b) garantía de accesibilidad y seguridad,

c) buenas condiciones de visibilidad,

d) posibilidad de construir infraestructura adecuada,

e) contar con todos los permisos correspondientes,

f) financiamiento,

g) planificación,

h) un staff preparado para la atención del público visitante y para la realización de visitas guiadas.

- Los sitios y entidades que gozan de este tipo de valoración codifican en la base de datos con el número 1 (uno).

- Los sitios no aptos para cumplir con fines educativos codifican con el número 0 (cero).

Valor turístico

Se refiere al potencial que presentan los recursos arqueológicos para generar emprendimientos turísticos en la actualidad. El objetivo central, en este caso, es la obtención de un rédito económico y se procura fomentar la difusión y el conocimiento por parte de la comunidad para lograr que el sitio o la localidad arqueológica sean visitados. En este caso, deben tomarse los recaudos necesarios para mantener su integridad y salvaguarda por medio de adecuados planes de manejo que sean elaborados y supervisados por arqueólogos capacitados en gestión patrimonial.

Aplica también para aquellas entidades susceptibles de ser manejadas con fines recreativos, de modo que el patrimonio se convierte en un recurso cultural que debe recibir "un manejo se- 
guro y adecuado" (Trotzig 1989:62) para que se preserve de la mejor manera posible. Pero, aún cuando la preservación de los sitios fuera óptima, es importante también que los visitantes vivan experiencias positivas y satisfactorias (Hall y Mc Arthur 1996), por lo que deberá contarse con equipos de especialistas en el tema que desarrollen planes de manejo eficaces.

- Los sitios y entidades que gozan de este tipo de valoración codifican en la base de datos con el número 1 (uno).

- Los sitios que no gocen de esta valoración codifican con el número 0 (cero).

Valor estético/paisajístico

Está constituido por una serie de atributos que tienen que ver exclusivamente con lo sensorial/perceptivo, y que hacen del bien arqueológico una entidad digna de protección por causa del goce que produce su simple contemplación, permanencia y conocimiento.

- Los sitios y entidades que gozan de este tipo de valoración codifican en la base de datos con el número 1 (uno).

- Los sitios que no gocen de esta valoración codifican con el número 0 (cero).

Valor político

El valor político estará determinado por la existencia de normas y/o declaratorias de protección legal específica. Esto conlleva a la creación de planes de manejo adecuados y a la puesta en valor de los sitios arqueológicos.

Otros

Otros tipos de valoración que se detecten en el futuro.

Si bien la variable "otros" resulta irrelevante al momento del uso de la herramienta digital, se considera importante dejar este campo abierto para incluir información que pudiera detectarse en relación a situaciones conflictivas (propias de todo tipo de relación social y valorativa) que ameriten, eventualmente, generar cambios en la PDM.

\section{Conclusiones}

Como resultado de este proceso planificado de recopilación de información, ahora se cuenta con un instrumento informático de síntesis que concentra todos los datos arqueológicos disponibles, cuantitativos y cualitativos, del área de interés que inicialmente comprendió los partidos de bonaerenses Azul, Tandil y Olavarría ${ }^{4}$

Una de las principales contribuciones del SIPAB es su capacidad de dar respuesta a las necesidades concretas y a las características específicas del registro arqueológico del área TOA. Por ello, el diagnóstico de situación y la caracterización histórica del paisaje de los tres partidos (en el sentido de Clark et al. 2004) resultaron fundamentales a la hora de iniciar el proceso de estructuración de los datos disponibles (Mariano 2012). De este modo, se puede disponer un cúmulo de información rápida y eficazmente, tanto a los fines de la gestión patrimonial como a los de la investigación.

A su vez, en función de los criterios de valoración detectados, fue posible establecer criterios de selección para las entidades arqueológicas listadas en el sistema. Así, por ejemplo, se pudo elaborar un ranking general teniendo en cuenta la cantidad de codificaciones positivas y negativas de cada sitio. De este modo, aquellos que tienen mayor significación 
(tanto desde el punto de vista científico como social) quedaron diferenciados de aquellos cuya valoración resultó menor. A la hora de realizar propuestas de gestión concreta de los recursos patrimoniales, este tipo de información es la más relevante.

También sería posible elaborar otro tipo de ranking, que atienda a variables tales como el estado de conservación o la vulnerabilidad de los sitios del TOA, por ejemplo.

Otra de las principales contribuciones de este sistema de información geográfico aplicado a la región, es que tiene en cuenta la significación social del patrimonio arqueológico, buscando diversificar las voces involucradas en los procesos de interpretación del pasado (Tully 2007:158).

De este modo es posible generar un conocimiento integral del patrimonio regional, que permita comprender su importancia científica y la apreciación comunitaria, a fin de identificar áreas sensibles y vulnerables, o evaluar posibilidades de cambio en cada contexto específico de gestión.

En este sentido, puede afirmarse que la gestión del patrimonio arqueológico requiere de un abordaje amplio y comprensivo de todos sus aspectos, así como de la participación democrática y de un manejo sustentable, para garantizar su preservación largo plazo.

La gestión de la información constituye el paso previo y la condición sine qua non para el desarrollo de mecanismos adecuados de manejo del patrimonio arqueológico en cualquier área de interés. Se espera, por lo tanto, que el SIPAB constituya un instrumento de gestión para la planificación de estrategias que atiendan a la salvaguarda y valoración social del patrimonio regional.

Agradecimientos: Estas investigaciones incluyen una parte de los resultados de la tesis doctoral de Carolina Mariano (2012), fueron realizadas en el marco de las investigaciones desarrolladas por PATRIMONIA (Estudios Interdisciplinarios de Patrimonio) de INCUAPA (Unidad Ejecutora CONICET-UNICEN) y financiadas por fondos provenientes de los proyectos PICT 0561/11 de la ANPCyT y PIP 429-2012 de CONICET, ambos dirigidos por la Dra. María Luz Endere, así como el PIP 349-2012 de CONICET, dirigido por la Dra. Victoria Pedrotta. Nuestro agradecimiento al Dr. Gustavo Politis, por sus valiosos comentarios y a los evaluadores del trabajo, cuyas observaciones y sugerencias permitieron mejorarlo. No obstante, todo lo expresado en el mismo es responsabilidad de las autoras.

\section{Notas}

1 En 1990 se definió a un SIG como"un sistema de hardware, software y procedimientos diseñados para facilitar la obtención, gestión, manipulación, análisis, modelación y salida de datos espacialmente referenciados, para resolver problemas complejos de planificación y gestión" (NCGIA 1990; Ladrón de Guevara 2011:35).

2 En el TOA puede localizarse, al menos, un tramo del llamado "Camino de los Chilenos", que conectaba los campos bonaerenses con Chile (Martínez Sierra 1975; Mandrini y Ortelli 1992). 3 En este caso, la lista de control propuesta no incluye geoformas que no correspondan al área de interés, pero podrían agregarse muchas otras que codificaran con otros números (i.e. montañas, valles de montañas, mesetas, dunas, médanos, costa marina, etcétera). 
4 Toda la información consignada en la base de datos se extrajo de información publicada y se incluyen las fuentes éditas de donde se extrajo. Se prevé en una segunda etapa incluir material gráfico así como apéndices digitales con las fuentes consultadas.

\section{Bibliografía citada}

Ballart Hernández, J. y M. A. Petit

1991 Un proyecto de formación en la gestión del patrimonio. Revista de Arqueología, 127: 12-15.

Bayón, C.; Pupio, A.; González, I.; Flegenheimer, N. y M. Frére

2007 Arqueología de las pampas. Sociedad Argentina de Antropología, Buenos Aires. 2 tomos.

Berón, M. y G. Politis.

1997 Arqueología Pampeana en la Década de los 90. Museo de Historia Natural de San Rafael e INCUAPA, Mendoza.

Berón, M.; L. Luna, C. Montalvo, C. Aranda y M. Carrera

2010 Mamül Mapu: pasado y presente desde la arqueología pampeana. Libros del Espinillo, Ayacucho.

Clark, J.; J. Darlington y G. Fairclough

2004 Using Historic Landscape Charcterisation. English Heritage. Lancashire Country Counscil, Londres.

Cleere, $\mathrm{H}$.

1993 Managing the archaeological heritage. Antiquity 67: 400-405.

Consejo de Europa.

2000 Convenio Europeo del Paisaje, Florencia, 20 de octubre de 2000. Sitio.web:http:/ / www. mma.es/secciones/desarrollo_territorial/paisaje_dt/convenio_paisaje/pdf/CONVENIO_EUROPEO_PAISAJE_Web.pdf

Curtoni, R.

2007 Arqueología y paisaje en el área centro-este de la provincia de La Pampa: la espacialidad humana y la formación de territorios. Tesis Doctoral inédita. Facultad de Ciencias Naturales y Museo, Universidad Nacional de La Plata, La Plata.

Endere, M. L.

2009 Algunas reflexiones acerca del patrimonio. Patrimonio, Ciencia y Comunidad. Su abordaje en los partidos de Azul, Olavarría y Tandil (ed. por M. L. Endere y J. L. Prado), pp. 17-46. INCUAPA, Universidad Nacional del Centro de la Provincia de Buenos Aires, Olavarría.

Endere, M. L. y J. L. Prado.

2009 Criterios de selección, valoración y zonificación de yacimientos arqueológicos y paleontológicos. Patrimonio, Ciencia y Comunidad. Un abordaje en los partidos de Azul, Olavarría y Tandil (ed. por M. L. Endere y J. L. Prado, 47-65. INCUAPA, Universidad Nacional del Centro de la Provincia de Buenos Aires, Olavarría. 
González Méndez, M.

2000 La revalorización del patrimonio arqueológico. La definición de un programa para el ayuntamiento de Toques (A. Coruña). Xunta de Galicia, España.

Gradin, C. y F. Oliva

2004 La región pampeana: su pasado arqueológico. Laborde, Rosario.

Guber, R.

2004 El salvaje metropolitano. Reconstrucción del conocimiento social en el trabajo de campo. Paidós, Buenos Aires.

Hall, M. y S. McArthur.

1996 The human dimension of Heritage Management: different values, different interests, different issues. Heritage management in Australia and New Zeland. The Human Dimension (ed. por M. Hall y S. McArthur), pp. 1-21. Oxford University Press, Oxford.

Ladrón de Guevara, B.

2011 Estándares mínimos de Registro del Patrimonio Arqueológico. Chile: Centro Nacional de Conservación y restauración DIBAM, Consejo de Monumentos Nacionales, área del Sistema Nacional de Coordinación de Información Territorial, Chile.

Ley Nacional 25.7432003 de Protección del Patrimonio Arqueológico y Paleontológico. Congreso Argentino de Buenos Aires, 25 de junio de 2003. Buenos Aires.

Mandrini, R. y S. Ortelli

1992 Volver al país de los araucanos. Sudamericana, Buenos Aires.

Martínez, G.; M. Gutiérrez; R. Curtoni; M. Berón y P. Madrid

2004 Aproximaciones Contemporáneas a la Arqueología Pampeana. Universidad Nacional del Centro de la Provincia de Buenos Aires, Olavarría.

Mariano, C.

2012 Herramientas teórico-metodológicas para la gestión sustentable del patrimonio arqueológico del centro de la provincia de Buenos Aires. Tesis Doctoral inédita. Facultad de Ciencias Sociales de la Universidad Nacional del Centro de la provincia de Buenos Aires, Olavarría.

Mariano, M.; Endere, M. L. y C. Mariano

2013 Herramientas metodológicas para la gestión del patrimonio intangible. El caso del partido de Olavarría, Buenos Aires, Argentina. Revista Colombiana de Antropología. En prensa.

Mark, S.

1996 Writing environmental and park histories. Heritage management in Australia and New Zeland. The Human Dimension (ed. por M. Hall y S. McArthur, pp. 153-159. Oxford University Press, Oxford.

Martínez Sierra, R.

1975 El mapa de las Pampas. Archivo General de la Nación Argentina, Buenos Aires. 
Mazzanti, D., Berón, M. A. y F. Oliva

2002 Del Mar a los Salitrales. Actas del II Congreso de Arqueología de la Región Pampeana Argentina. Universidad Nacional de Mar del Plata, Mar del Plata.

National Center for Geographic Information and Analysis.

1990 National Center for Geographic Information and Analysis. Santa Barbara: Universidad de California.

Paz, C.

2009 El desarrollo de la minería en el partido de Olavarría. Patrimonio, ciencia y comunidad. Su abordaje en los partidos de Tandil, Olavarría y Azul (ed. por M. L. Endere y J. L. Prado), pp. 283-302. Universidad Nacional del Centro de la Provincia de Buenos Aires, Olavarría.

2012 Prácticas productivas de los italianos en el partido de Olavarría. La incidencia de la inmigración italiana en la transferencia de técnicas y tecnologías para la minería de la cal y del granito en las sierras olavarrienses (1880-1920). Tesis Doctoral inédita. Universidad de Buenos Aires. Facultad de Filosofía y Letras, Buenos Aires.

Pedrotta, V.

2005 Las sociedades indígenas de la provincia de Buenos Aires entre los siglos XVI y XIX. Tesis doctoral inédita. Facultad de Ciencias Naturales y Museo de la Universidad Nacional de La Plata, La Plata.

2009 Algunas consideraciones en torno al valor patrimonial y a la preservación de las construcciones de piedra del Sistema de Tandilia. Patrimonio, ciencia y comunidad. Su abordaje en los partidos de Azul, Olavarría y Tandil (ed. por M. L. Endere y J. L. Prado), pp. 205-230. Universidad Nacional del Centro de la Provincia de Buenos Aires, Olavarría.

Politis, G. y P. Madrid

2001 Arqueología pampeana: estado actual y perspectivas. Historia Argentina Prehispánica (ed. por E. Berberián y A. Nielsen), pp. 737-814. Brujas, Buenos Aires.

Planning Policy Guidance (PPG 16)

2006 Note 16. www.english-heritage.org.uk/.../archaeological-scien.UK. (15 diciembre 2013).

Trombold, C.

1991 Ancient road networks and settlements hierarchies in the New World. Cambridge University Press, Cambridge.

Trotzig, G.

1989 The cultural dimension of development, an archaeological approach. Archaeological Heritage Management in the Modern World (ed. por H. Cleere), 59-63. Council of British Archaeology, Londres.

Tully, G.

2007 Community Archaeology: general methods and standards of practice. Public Archaeology 6 (3) 155-187. 